\author{
Mary Giachnaki ${ }^{1}$, Elena Athanasiadi ${ }^{1}$, \\ Abraham Pouliakis $^{1 \star}$, Aris Spathis ${ }^{1}$, \\ Christine Kottaridi ${ }^{1}$, Evangelia Aga ${ }^{1}$, \\ Maria Papaefthimiou ${ }^{2}$, Panagiota \\ Mentzelopoulou ${ }^{2}$, Helen Spathi ${ }^{3}$ and \\ Petros Karakitsos $^{1}$ \\ ${ }^{1}$ Department of Cytopathology, National and \\ Kapodistrian University of Athens, Athens, Greece \\ ${ }^{2}$ Department of Cytopathology, "Alexandra" \\ Hospital, Athens, Greece \\ ${ }^{3}$ Department of Cytopathology, "Agia Olga" \\ Hospital, Athens, Greece \\ Dates: Received: 22 December, 2015; Accepted: \\ 27 February, 2016; Published: 28 February, 2016 \\ *Corresponding author: Dr. A Pouliakis, Department \\ of Cytopathology, National and Kapodistrian \\ University of Athens, ATTIKON University Hospital \\ Rimini 1, Haidari, 12462, Athens, Greece, Tel: \\ +30 210 5831948; +30 6972846977; Fax: +30 210 \\ 5831942; E-mail: apouliak@med.uoa.gr; apou1967@ \\ gmail.com \\ www.peertechz.com
}

Keywords: Cytopathology; Cytology; Cervical cytology; Test Papanicolaou; Liquid based cytology; Thin Prep; Cost effectiveness; Molecular techniques

\section{Research Article \\ Comparative Analysis of Conventional and Thin Prep Papanicolaou Test. Technical and Economic Aspects}

\begin{abstract}
Abbreviations
CxCa: Cervical Cancer; LBC: Liquid Based Cytology; ASCUS: Atypical Squamous Cells of Undetermined Significance; AGUS: Atypical Glandular Cells of Undetermined Significance; LSIL: Low grade Squamous Intraepithelial Lesion; HSIL: High grade Squamous Intraepithelial Lesion; NASBA: Nucleic Acid Sequence Based Amplification; HPV: Human Papilloma Virus; CIN: Cervical Intraepithelial Neoplasia; YoLS: Years of Life Saved; ICC: Immunocytochemistry; CC: Conventional Cytology; IVD: In Vitro Diagnostics; CEA: Cost Effectiveness Analysis;

\section{Background}

Cervical cancer $(\mathrm{CxCa})$ is the fourth most common cancer in women (after breast, colorectal, and lung cancers) and a leading cause of cancer death in females worldwide [1]. More than $85 \%$ of these cases and deaths are in developing countries; this is due to lack of screening that may allow detection of precancerous and early stage cervical cancer. Despite the advances in screening, cervical cancer remains a serious problem of public health even in developed countries, due to detection failures [2].
\end{abstract}

Since the introduction of cytopathology in 1928 [3] and the application of the popular Papanicolaou test (test Pap) [4,5] the method of sampling, preparation and evaluation of conventional cervicovaginal smears has not changed drastically. The combination of the low cost and the high levels of diagnostic accuracy contributed to the method popularity. Scientific data indicate that the periodic examination of women using Papanicolaou test leads to a reduction of mortality from cervical cancer by $70 \%$.

ThinPrep Pap test is a Liquid Based Cytology (LBC) method, representing the first, after 50 years, evolution of classical Papanicolaou test (Conventional Cytology - CC). This method initiates changes in the way of fixation and production of slides which enhance dramatically the smear quality. Due to this reason, ThinPrep Pap test was authorized in 1996 by the Food and Drug Administration (FDA) of U.S.A. as a replacement to the conventional Papanicolaou test. In ThinPrep, the smears, instead of layering on the glass slides immediately after their extraction form the cervix, they are collected using a sampling device which is rinsed into a vial containing a fixative solution (PreserveCyt). The vial is then transported to the cytopathology laboratory where a slide is prepared by specialized modalities that create a single layer of cells on the slide with total area less than $50 \%$ comparing to the area of a conventional slide. The remaining biological material in the LBC vial can be used for molecular techniques. 
The cytological findings, irrelevant of the applied method (CC or LBC), are nowadays reported and formulated according to the revised Bethesda classification system (TBS2001 system) [6,7]. The management of women according to the diagnostic categories proposed by the TBS2001 is:

1. Inadequate: in this case the Papanicolaou test should be repeated.

2. WNL (Within Normal Limits): No clinical approach is required, the test should be repeated after a few years (three of five according to the applied national strategy)

3. ASCUS (Atypical Squamous Cells of Undetermined Significance) or AGUS (Atypical Glandular Cells of Undetermined Significance): The woman is requested to perform colposcopy (or cytological examination after a period).

4. LSIL (Low grade Squamous Intraepithelial Lesion): The woman is requested to repeat Papanicolaou test. According to the scientific literature $25 \%$ of LSIL cases progresses to HSIL, $25 \%$ progresses to cancer and $50 \%$ regress, as this procedure takes many years.

5. HSIL (High grade Squamous Intraepithelial Lesion): The woman should be treated; the applied surgical treatment is conization. After therapy the survival rate is similar to that of healthy women.

6. Cancer cases: surgical treatment is performed, followed by radiotherapy; the expected survival is 5 years.

$\mathrm{CxCa}$ is known to be caused almost always by human papillomavirus (HPV) infection which is the commonest sexually transmitted infection worldwide. There are about 100 types of HPV virus that can infect humans. Among them, 15 are oncogenic and may cause CxCa. The improved understanding of HPV infection along with the natural history of cervical neoplasias have nowadays, resulted in the addition of the HPV DNA test along with the Papanicolaou test as ancillary test and frequently reported as a competing test. In summary, tests related to HPV lifecycle include HPV DNA typing or identification of the existence of high risk subtypes, mRNA identification of the viral E6/E7 oncogenes that are linked to oncogenic activation and immunocytochemical examinations. LBC is provided the means to perform these additional examinations.

LBC is the most widely used starting material for the detection of HPV DNA, since nucleic acid preservation is far superior to conventional cytology samples. Unfortunately, the overall accuracy for HPV detection varies greatly depending on the primer set, the reaction conditions and the enzyme used. PCR based techniques have high sensitivity, but usually suffer from false positives due to crosscontaminations and miss-priming. Specifically concerning HPVs, there were two strategies used; one that utilizes primers that are type specific resulting in increased specificity and one that uses primers that are designed for well conserved sequences of a target gene, usually L1, resulting in amplification of various HPV types with a single primer set. The latter has been widely used in detecting HPVs in cervical samples and has provided clinical evidence of the connection of HPVs with cervical cancer. More recently developed tests for HPV detection have started using the Real-time PCR platforms. Compared to conventional PCR, Real-time PCR has many advantages such as the lower detection limit, due to increased sensitivity, and the ability to use several chemistries that allow superb specificity.

One of the significant advantages of LBC is, that due to the presence of alcohols, mRNAs are adequately preserved [8]. Detection of mRNAs of the oncogenic products of HPV E6 and E7 [9], have been studied in order to identify women with higher risk to develop HSIL. From the various methods NASBA (Nucleic Acid Sequence Based Amplification) has been shown to be more specific than DNA test, more effective in identifying HSILs after treatment than repeat cytology and more accurate in identifying women with HSILs, thus reducing revisits and referral colposcopies [10-12]. A similar amplification method used by a commercial test (APTIMA HPV Assay, Gen-Probe, U.S.A.) has produced results that show strong correlation of a positive result with severity of the lesion [13], with more recent studies supporting that Aptima had similar sensitivity to HC2 with improved specificity [14]. However, others have found poor specificity [15]. More recently, a flow cytometry based assay (HPV Noncontact, InCellDx, U.S.A.) has been compared to HPV DNA testing versus Hybrid Capture 2 and versus CLART2 typing, with similar sensitivity and greater specificity/positive predictive value than HPV DNA testing [16-18]. Furthermore, flow cytometry allows discrimination and quantification of the cellular populations present in the cervical sample that in turn allows the characterization of sample adequacy $[19,20]$.

Due to the residual material in the LBC vials, it is possible to prepare multiple slides per biological sample. This has allowed the use of immunocytochemistry (ICC). The qualitative detection of the p16 ${ }^{\mathrm{INK} 4 \mathrm{~A}}$ protein in cervical cytology preparation from LBCs, is one of such supplementary techniques. This ICC method has been used in the identification of women with positive high risk HPV test results or women with high grade cervical intraepithelial lesions in screening populations. In CIN, p16 $6^{\mathrm{INK} 4 \mathrm{~A}}$ has been shown to be overexpressed after the inactivation of $\mathrm{pRb}$ mediated by the E7 oncoprotein from high risk types of HPV. The overexpression of $\mathrm{p} 16^{\mathrm{INK} 4 \mathrm{~A}}$ protein is directly linked to the oncogenesis and thus has been proposed as a future biomarker. The protein expression of p16 $6^{\mathrm{INK} 4 \mathrm{~A}}$ combined with Ki67, as a commercial KIT (CINtec Plus, Roche, Switzerland), has been used to identify, with significant higher specificity to DNA detection, women with HSIL or with higher risk to develop HSIL [2123].

As the performance of each screening method (CC or LBC) differs, and the associated management costs are important, the comparison of the two methods should be in multiple levels, the technical level, which addresses only performance issues and a financial level that takes into account the various associated costs. In this study we evaluate the performance of both methods in a setting involving three Greek hospitals, and carry out the cost effectiveness analysis (CEA). In addition we summarize the pros and cons of the two methods. To the authors' knowledge this is the first study in the 
Greek health care environment.

\section{Materials and Methods}

\section{Study population}

In order to compare the performance of the CC and the LBC, 23,604 conventional smears from the same number of women were analysed and 23,604 ThinPrep smears from equal number of different women. The smears were collected from 1/2/2006 until 31/1/2007.

The study was performed at the departments of cytopathology of a) University General Hospital ATTIKON b) "Alexandra" Hospital and c) "Agia Olga" Hospital, all located in Athens, Greece. The study was conformant to the Helsinki declaration; as there are no interventions in the participating women, there was no requirement to obtain signed informed consent forms from the study population.

\section{Methods}

The cytological findings were formulated according to the revised Bethesda classification system (TBS2001 system) [6,7]. We considered that the standard clinical approach is applied, according to each diagnostic category as presented in the background section.

In order to address the cost of the various examinations and treatments, we used the costs as suggested by the Greek National Health System, specifically:

Cost of conventional Papanicolaou test: Includes the cost of woman admission, consumables, the health professional time costs, and the time required to be spent by the woman (about 4 hours). This cost was: $15.99 €$.

Cost of ThinPrep Pap test: In this case the required consumables are different, due to the use of a vial containing the liquid and the application of a technique to create single layer specimens, which requires the use of an additional filter by a device (ThinPrep Processor: TP2000). The depreciation percentage equals to $20 \%$ of the device price per year. The cost of ThinPrep Pap test was calculated to be $25.94 €$, including depreciation.

Cost of treatment: The price that insurance organizations reimburse the hospital was used as treatment cost. This cost is for colposcopy: $11.74 €$, for conization $103.00 €$ and for cancer therapy (surgical treatment, medication and radiotherapy) $3,276.4 €$ (mean value).

Assessment: In order to assess the results with orientation to the outcome of the health status of women and the effectiveness of diagnosis, two parameters were considered: the calculation of years of life saved (YoLS) and the Cost to "win" a single YoLS (Cost/YoLS), being the fraction of the additional cost required for ThinPrep Pap test by the total years of life gained in the study population.

For the calculation of the YoLS, we considered the life expectancy of Greek women as it is reported by the National Statistics Institute, which is 80.7 years. As the average age of women in this study is 55 years and the life expectancy of women with $\mathrm{CxCa}$ is 5 years, the YoLS for every woman diagnosed at an early stage of the disease and therefore does not progress to cancer are 20.7 years.

\section{Results}

The results of the CEA are summarized in table 1 . Initially we calculated the cost of the first round examinations for CC and LBC, this was $377,427.96 €$ and $612,287.76 €$ respectively (i.e. $234,859.80$ $€$ higher cost for LBC). Subsequently we added the cost required to repeat examinations due to inadequate samples. Specifically, with CC it was required to repeat 5,556 tests, while via LBC 3,456 more tests. This increased the cost of LBC by $808.20 €$.

However, due to the higher performance of ThinPrep Pap test, there were improved health outcomes, specifically:

- 597 more cases of ASCUS and AGUS had to undergo colposcopy as CC resulted in 1,134 such cases and ThinPrep cytology in 537 (table 1). These women, according to the applied policy, had to be examined via colposcopy, this had an increased cost in CC by $7,008.78 €$ (which is counted as benefit of LBC and thus is subtracted)

- ThinPrep enabled monitoring of 135 (621 - 486) more cases of LSIL which were not detected by CC. Those women had to be called again to repeat test Papanicolaou. This results in $8,337.60 €$ additional cost of LBC (as test Papanicolaou via LBC costs about $10 €$ higher than CC)

- For 21 (132-11) CC missed to detect a HSIL, these women are referred to colposcopy (cost $103.00 € /$ case), this increased the cost of LBC by $2,163.00 €$.

- Finally there were no differences in the number of detected Ca cases, 51 cases which had to be treated (cost 3,276.40€/ case).

Summarizing at this stage (see table 1 ) the cost of interventions for CC was 665,882.10€ while for LBC 905,041.92€, thus LBC had an overhead of $239,159.82 €$ for the same number of women $(23,604)$.

However the lower performance of CC resulted in:

- For 34 women (33.75 in table 1) that a non-detected LSIL lesion will progress to $\mathrm{Ca}$, these patients require surgical treatment, medication and radiotherapy, the associated cost is $110,578.50 €$ which could be avoided by LBC

- Similarly there are 34 women (33.75 in table 1 ) that a nondetected LSIL lesion will progress to HSIL due to detection failure in CC. The associated cost is $3,476.25 €$ as these women had to referred for colposcopy

- Finally there are 21 cases of HSIL missed by CC, these is expected to progress to $\mathrm{Ca}$, the associated treatment cost is $68,804.40 €$.

In summary, the cost of the detection failures (loses due to missed cases in table 1) is $182,859.15 €$ against CC.

By summarizing the additional cost of LBC and the cost of detection failures in $\mathrm{CC}$ total cost of diagnostic and therapeutic actions for the studied cases due to ThinPrep Pap test is higher by $56,300.67 €$ for the 23,604 women. However the YoLS for the 55 (54.75) cases of cancer that were prevented was 1133.33 years [ 54.75 women * 20.7 years/woman]. Thus the cost per saved life year (Cost/YoLS) 
Table 1: Cost Effectiveness Analysis of conventional cytology and ThinPrep cytology.

\begin{tabular}{|c|c|c|c|}
\hline & $\begin{array}{l}\text { Conventional } \\
\text { Cytology }\end{array}$ & ThinPrep Cytology & $\begin{array}{l}\text { Additional cost of ThinPrep vs. } \\
\text { Conventional }\end{array}$ \\
\hline Number of women & 23,604 & 23,604 & \\
\hline First round examinations & 23,604 & 23,604 & \\
\hline Cost of first round examinations & $377,427.96 €$ & $612,287.76 €$ & $234,859.80 €$ \\
\hline Number of repeated examinations & 5,556 & 3,456 & \\
\hline Cost of repeated examinations & $88,840.44 €$ & $89,648.64 €$ & $808.20 €$ \\
\hline Subtotal cost & & & $235,668.00 €$ \\
\hline Number of ASCUS-AGUS cases & 1,134 & 537 & \\
\hline Percentage of ambiguous cases (ASCUS-AGUS) & $4.80 \%$ & $2.28 \%$ & \\
\hline Cost for each ASCUS-AGUS case (colposcopy) & $11.74 €$ & $11.74 €$ & \\
\hline Subtotal cost & & & $228,659.22 €$ \\
\hline Number of LSIL cases & 486 & 621 & \\
\hline Percentage of LSIL cases & $2.06 \%$ & $2.63 \%$ & \\
\hline Cost for each LSIL case (repeat Papanicolaou test) & $15.99 €$ & $25.94 €$ & \\
\hline Cost for LSIL cases & $7,771.14 €$ & $16,108.74$ & $8,337.60 €$ \\
\hline Subtotal cost & & & $236,996.82 €$ \\
\hline Number of HSILcases & 111 & 132 & \\
\hline Percentage of HSIL cases & $0.47 \%$ & $0.56 \%$ & \\
\hline Cost for each HSIL case (conization) & $103.00 €$ & $103.00 €$ & \\
\hline Cost for HSIL cases & $11,433.00 €$ & $13,596.00 €$ & $2,163.00 €$ \\
\hline Subtotal cost & & & $239,159.82 €$ \\
\hline Number of Ca cases & 51 & 51 & \\
\hline Cost for each Ca case (surgical treatment, medication and radiotherapy) & $3,276.40 €$ & $3,276.40 €$ & \\
\hline Cost for Ca cases & $167,096.40 €$ & $167,096.40 €$ & $0.00 €$ \\
\hline Cost of interventions & $665,882.10 €$ & $905,041.92 €$ & $239,159.82 €$ \\
\hline Cases of Ca cases missed as LSIL & 33.75 & 0 & \\
\hline Cost for each Ca case (surgical treatment, medication and radiotherapy) & $3,276.40 €$ & 3276.4 & \\
\hline Cost of missed cases & $110,578.50 €$ & $0.00 €$ & $-110,578.50 €$ \\
\hline Cases of HSIL missed as LSIL & 33.75 & 0 & \\
\hline Cost for each HSIL case (conization) & $103.00 €$ & $103.00 €$ & \\
\hline Cost of missed cases & $3,476.25 €$ & $0.00 €$ & $-3,476.25 €$ \\
\hline Cases of Ca missed as HSIL & 21 & 0 & \\
\hline Cost for each Ca case (surgical treatment, medication and radiotherapy) & $3,276.40 €$ & $3,276.40 €$ & \\
\hline Cost of missed cases & $68,804.40 €$ & $0.00 €$ & $-68,804.40 €$ \\
\hline Loses due to missed cases & $182,859.15 €$ & $0.00 €$ & $-182,859.15 €$ \\
\hline Total cost (includes cost for examinations and treatment of missed cases) & $848,741.25 €$ & $905,041.92 €$ & $56,300.67 €$ \\
\hline Number of additional cases that detected and will not progress to cancer & & 54.75 & \\
\hline YoLS per cancer case & & 20.7 & \\
\hline YoLS & & 1133.33 & \\
\hline Cost/YoLS & & $49.68 €$ & \\
\hline
\end{tabular}


is $49.68 €$. This cost is significantly lower that the cut off proposed by the insurance organizations, which in the U.S.A, for instance, is in the range of $50,000 \$$.

There are several other aspects of ThinPrep cytology and in general LBC that are not considered in the cost effectiveness analysis in this study, most of them would result in even lower cost/YoLS:

1. The consumed time by cytopathologists: The average time to examine a single ThinPrep slide is 3 minutes, while the average examination time for conventional slides is 6 minutes. In the sample studied, the LBC method leaded to a reduction of 1581 man hours.

2. As ThinPrep vial allows additional examinations without the need for an additional sample, there is no need to recall women; this produces additional cost savings for the patients.

3. Transportation of samples from sampling sites to the cytopathology laboratory facilities via LBC reduces errors and contributes to the reduction of pre-analytical errors.

4. The shorter turnaround time in the cytopathology laboratory and the less time needed to obtain the examination results along with the higher sensitivity comparing to conventional cytology contribute towards the confidence of the method and a higher trust level on the patient side.

5. The higher confidence level contributes as well to the reduction of the number of women who do not follow or quit the organized screening programs.

6. The LBC method introduces one more stage in the slide preparation, i.e. the use of a device that creates mono-layer slides; this process introduces an overhead of about one minute for slide preparation. This overhead is not considered in the study, however to the authors' opinion this may be surpassed as the overhead for the cytopathologists for conventional slides is about three minutes/specimen.

7. LBC vial has higher weight and volume than conventional cytology slides, thus transportation costs for the same number of samples is higher. This cost was not considered in this analysis, as samples are transferred by sample takers (midwifes) to the cytopathology laboratory on a scheduled basis. On every visit, sample takers receive the cytological results of the previous batch and submit the new samples. Additionally, many of the samples are obtained from the hospital clinics, thus there is no associated transportation cost. In the authors' opinion, additional cost for sample transportation would add a relatively small cost to the process compared to the health outcome gains.

8. Other advantages of LBC include the alcohol of the solutions which acts as a fixative for cells, inactivates microbial flora that causes lyses or red blood cells and mucus. Additionally, the $\mathrm{pH}$ of the solution allows the preservation of the morphological characteristics of the cells. Moreover, via LBC method, all collected cells are removed from the collection device immediately, thus dehydration and oxidization of cells are avoided as they remain exposed in the air for a very short time. The availability of the biological material in the vial allows the application of ancillary molecular methods.

The results of this study indicate significant performance improvements, specifically the percentage of inadequate samples was reduced from $23.54 \%$ to $14.64 \%$ (37.8\% improvement), which is a statistically significant difference $(8.90 \%, 95 \%$ CI: $8.19 \%$ to $9.61 \%$, $\mathrm{x}^{2}=604.66 \mathrm{p}<0.0001$ ). The number of ambiguous cases (ASCUS and AGUS) was reduced from 1,134 to 537 (4.80\% and $2.28 \%$ respectively, as percentage of all cases), this is $52.6 \%$ improvement, while the difference in the proportions is statistically significant (difference $=2.52 \%, 95 \%$ CI: $2.19 \%$ to $2.86 \%, \mathrm{x}^{2}=218.75, \mathrm{p}<0.0001$ ). There were detected 135 more LSIL cases (percentage $2.06 \%$ and $2.63 \%$ for CC and LBC respectively, on the basis of the 23,604 studied cases), the improvement is $27.7 \%$, while the difference in the two percentages is statistically significant (Difference $=0.57 \%, 95 \%$ CI: $0.29 \%$ to $0.85 \%$, $\left.\mathrm{x}^{2}=16.50, \mathrm{p}<0.0001\right)$. Finally LBC detected 21 additional HSIL cases that were not detected by CC (132 vs. 111 cases, $0.56 \%$ and $0.47 \%$ respectively), the improvement in the detection rate of HSIL is $19.1 \%$ while there is no statistically significant difference between the two percentages (Difference $=0.09 \%, 95 \%$ CI: $-0.04 \%$ to $0.22 \%, \mathrm{x}^{2}=1.69, \mathrm{p}$ $=0.1930)$. These results are in line with other studies [24,25], where the use of LBC (SurePath ${ }^{\mathrm{TM}}$ method, TriPath ${ }^{\mathrm{TM}}$, Burlington, NC), showed improvement in LSIL detection by $196 \%$ and HSIL by $243 \%$, such findings in these studies extend to carcinomas as well [25].

\section{Discussion and Conclusions}

According to published research, false positive rates in conventional Papanicolaou test range from 5-10\%. However the most severe issue remains the percentage of false negative results. According to bibliographical data, false negative cases range from $5 \%$ to $50 \%$, according to Gay et al. [26], this percentage is $20 \%$, and in a meta-analysis study performed by the Agency for Health Care Policy Research published in 1999, this percentage was 50\% [27]. Thus, quality control of the process and of cytopathology laboratories is an important issue [28-30], as it can increase the performance of the cytological examination.

Most of the studies agree that $60 \%-90 \%$ of false negative cases are due to wrong sampling of the biological material [26,31]. According to Hutchinson et al. [32], more than $80 \%$ of collected cells from the cervix are not deposited in the glass slides. Moreover, it is not possible for gynecologists to select a representative sample for the slide. This was evident in our analysis, as the percentage of inadequate samples was $23.54 \%$ and $14.64 \%$ for CC and LBC respectively, thus LBC reduced significantly $(\mathrm{p}<0.0001)$ the inadequate percentage by $38 \%$. The process for conventional slides often leads to bad quality specimens due to inadequate fixation and excess blood and mucus. Thus, the microscopic examination becomes laborious, difficult and error prone as it is hard to identify rare cells indicative of neoplasias. LBC clears all these artifacts and cytopathologists examine a smaller and clearer area of the slide.

In terms of ambiguous results (ASCUS and AGUS), the outcomes of this study showed a reduction of the relevant percentage $(4.80 \%$ and $2.28 \%$ ambiguous cases in CC and LBC respectively) by $53 \%$ 
$(\mathrm{p}<0.0001)$. Reduction of this percentage were reported in other studies, for example $26.59 \%$ was reported in [33] and $52.75 \%$ in [34].

Simulation experiments [35], showed that simply substituting CC via $\mathrm{LBC}$ without any change in compliance, may result in more than $32 \%$ in reductions of the incidence of $\mathrm{CxCa}$. It has been proved that LBC is a cost effective method when applied in the general population, specifically in the Greek health system the introduction of LBC costs less than $50 €$ per saved life year, a cost lower than the cut off used (about 50,000\$).

The cost effectiveness analysis subjects to numerous parameters, such as the incidence of the disease, the specific costs of the involved "components" including examination and treatment costs, the conformance of the population in the required repetitive process. All of these may be different from country to country and even in different regions in the same country. Future studies may include more detailed analysis for factors not being considered, for example savings due to the reduced work hours of cytopathologists and the economic effects on the women due to reduced psychological effects.

Conventional Papanicolaou test has saved and continues to save millions of women. The introduction of LBC 50 later was an important enhancement, in terms of performance as shown in this study and other existing studies, as well as for improved quality and enhanced standardization of the process. Moreover, LBC has paved the way for the application of modern molecular techniques, either as adjunctive to the test or claiming to be alternative. These modern techniques that would not be possible without $\mathrm{LBC}$, have the potential to provide important outcomes for the HPV lifecycle and the deeper knowledge of the disease's natural history.

\section{References}

1. World Health Organisation (2013) PRESS RELEASE $N^{\circ}$ 223: Latest world cancer statistics Global cancer burden rises to 14.1 million new cases in 2012: Marked increase in breast cancers must be addressed [Press release].

2. Leyden WA, Manos MM, Geiger AM, Weinmann S, Mouchawar J, et al (2005) Cervical cancer in women with comprehensive health care access: attributable factors in the screening process. J Natl Cancer Inst 97: 675-683.

3. Papanicolaou GN (1973) New Cancer Diagnosis. Paper presented at the 3rd Race Betterment Conference, Battle Creek 23: 174-179.

4. Papanicolaou GN, Traut HF (1997) The diagnostic value of vaginal smears in carcinoma of the uterus. 1941. Arch Pathol Lab Med 121: 211-224.

5. Diamantis A, Magiorkinis E, Koutselini $H$ (2014) 50 years after the death of George Nicholas Papanicolaou (1883-1962): evaluation of his scientific work. Acta Med Hist Adriat 12: 181-188.

6. Henry MR (2003) The Bethesda System 2001: an update of new terminology for gynecologic cytology. Clin Lab Med 23: 585-603.

7. Smith JH (2002) Bethesda 2001. Cytopathology 13: 4-10.

8. Srinivasan M, Sedmak D, Jewell S (2002) Effect of fixatives and tissue processing on the content and integrity of nucleic acids. Am J Pathol 161: 1961-1971.

9. Scheffner M, Whitaker NJ (2003) Human papillomavirus-induced carcinogenesis and the ubiquitin-proteasome system. Seminars in cancer biology 13: 59-67.

10. Molden T, Nygård JF, Kraus I, Karlsen F, Nygård M, et al. (2005) Predicting CIN2+ when detecting HPV mRNA and DNA by PreTect HPV-proofer and consensus PCR: A 2-year follow-up of women with ASCUS or LSIL Pap smear. International journal of gynecological cancer 114: 973-976.
11. Sorbye SW, Arbyn M, Fismen S, Gutteberg TJ, Mortensen ES (2011) HPV E6/E7 mRNA testing is more specific than cytology in post-colposcopy followup of women with negative cervical biopsy. PloS one 6: e26022.

12. Sorbye SW, Fismen S, Gutteberg T, Mortensen ES (2010) Triage of women with minor cervical lesions: data suggesting a "test and treat" approach for HPV E6/E7 mRNA testing. PLoS One 5: e12724.

13. Castle PE, Dockter J, Giachetti C, Garcia FA, McCormick MK, et al. (2007) A cross-sectional study of a prototype carcinogenic human papillomavirus E6/ E7 messenger RNA assay for detection of cervical precancer and cancer. Clin Cancer Res 13: 2599-2605.

14. Arbyn M, Roelens J, Cuschieri K, Cuzick J, Szarewski A, et al. (2013) The APTIMA HPV assay versus the Hybrid Capture 2 test in triage of women with ASC-US or LSIL cervical cytology: a meta-analysis of the diagnostic accuracy. Int J cancer 132: 101-108.

15. Szarewski A, Ambroisine L, Cadman L, Austin J, Ho L, et al. (2008) Comparison of predictors for high-grade cervical intraepithelial neoplasia in women with abnormal smears. Cancer epidemiology. biomarkers \& prevention 17: 3033-3042.

16. Coquillard G, Palao B, Patterson BK (2011) Quantification of intracellular HPV E6/E7 mRNA expression increases the specificity and positive predictive value of cervical cancer screening compared to HPV DNA. Gynecologic Oncology 120: 89-93.

17. Kottaridi C, Tsiodras S, Spathis A, Chranioti A, Pappas A, et al. (2011) Clinical performance of human papillomavirus E6, E7 mRNA flow cytometric assay compared to human papillomavirus DNA typing. Analytical and Quantitative Cytology and Histology 33: 305-310.

18. Spathis A, Kottaridi C, Chranioti A, Meristoudis C, Chrelias C, et al. (2012) mRNA and DNA detection of human papillomaviruses in women of all ages attending two colposcopy clinics. PloS one 7: e49205.

19. Kottaridi C, Georgoulakis J, Kassanos D, Pappas A, Spathis A, et al. (2010) Use of flow cytometry as a quality control device for liquid-based cervical cytology specimens. Cytometry. Part B, Clinical cytometry. 78: 37-40.

20. Polina R, Sturgis C, Patterson J, Patterson BK (2008) Rapid, high throughput determination of cervical cytology specimen adequacy using a capillarybased cytometer. Cytometry. Part B, Clinical cytometry. 74: 133-136.

21. Bergeron C, Ronco G, Reuschenbach M, Wentzensen N, Arbyn M, et al. (2015) The clinical impact of using p16(INK4a) immunochemistry in cervical histopathology and cytology: an update of recent developments. Int J Cancer 136: $2741-2751$

22. Capobianco G, Marras V, Wenger JM, Santeufemia DA, Ambrosini G, et al. (2013) P16 immunostaining and HPV testing in histological specimens from the uterine cervix. Eur J Gynaecol Oncol 34: 227-230.

23. Wentzensen N, Schwartz L, Zuna RE, Smith K, Mathews C, et al. (2012) Performance of p16/Ki-67 immunostaining to detect cervical cancer precursors in a colposcopy referral population. Clin Cancer Res 18: 41544162.

24. Sass MA (2004) Use of a liquid-based, thin-layer Pap test in a community hospital. Impact on cytology performance and productivity. Acta Cytol 48: 1722.

25. Fremont-Smith M, Marino J, Griffin B, Spencer L, Bolick D (2004) Comparison of the SurePath liquid-based Papanicolaou smear with the conventional Papanicolaou smear in a multisite direct-to-vial study. Cancer 102: 269-279.

26. Gay JD, Donaldson LD, Goellner JR (1985) False-negative results in cervical cytologic studies. Acta Cytol 29: 1043-1046.

27. McCrory DC, Matchar DB, Bastian L, Datta S, Hasselblad V, et al. (1999) Evaluation of cervical cytology. Evid Rep Technol Assess (Summ) 1-6.

28. Pouliakis A, Margari N, Spathis A, Kottaridi C, Stamouli M, et al. (2014) ISO 15189:2012 Technical Requirements for Cytopathology Laboratory Information Systems. International Journal of Reliable and Quality E-Healthcare (IJRQEH) 3: 58-80. 
29. Pouliakis A, Athanasiadi E, Karakitsou E, Archondakis S, Mourtzikou A, et al. (2014) ISO 15189:2012 Management Requirements for Cytopathology Laboratory Information Systems. International Journal of Reliable and Quality E-Healthcare (IJRQEH) 3: 37-57.

30. Niki M, Abraham P, Stavros A, Magdalini S, Dionysios A, et al. (2014) A Quality Control Study of Liquid-Based Cytology Test Papanicolaou: Design and Implementation Aspects of Laboratory Information Systems for Continuous Quality Control. International Journal of Reliable and Quality E-Healthcare (IJRQEH) 3: 1-21.

31. Joseph MG, Cragg F, Wright VC, Kontozoglou TE, Downing P, et al. (1991) Cyto-histological correlates in a colposcopic clinic: a 1-year prospective study. Diagn Cytopathol 7: 477-481.
32. Hutchinson ML, Isenstein LM, Goodman A, Hurley AA, Douglass KL, et al. (1994) Homogeneous sampling accounts for the increased diagnostic accuracy using the ThinPrep Processor. Am J Clin Pathol 101: 215-219.

33. Papillo JL, Zarka MA, St John TL (1998) Evaluation of the ThinPrep Pap test in clinical practice. A seven-month, 16,314-case experience in northern Vermont. Acta Cytol 42: 203-208.

34. Yeoh GP, Chan KW, Lauder I, Lam MB (1999) Evaluation of the ThinPrep Papanicolaou test in clinical practice: 6-month study of 16,541 cases with histological correlation in 220 cases. Hong Kong Med J 5: 233-239.

35. Montz FJ, Farber FL, Bristow RE, Cornelison T (2001) Impact of increasing Papanicolaou test sensitivity and compliance: a modeled cost and outcomes analysis. Obstet Gynecol 97: 781-788.

Copyright: () 2016 Giachnaki M, et al. This is an open-access article distributed under the terms of the Creative Commons Attribution License, which permits unrestricted use, distribution, and reproduction in any medium, provided the original author and source are credited. 